\title{
Clinical and Antibacterial Effects of Tualang Honey on Pseudomonas-induced Keratitis in Rabbit Eyes
}

\author{
Rajendran Punitan ${ }^{1}$, Siti Amrah Sulaiman ${ }^{2}$, Habsah B. Hasan ${ }^{3}$, Ismail Shatriah ${ }^{1}$ \\ 1. Ophthalmology, School of Medical Sciences, Universiti Sains Malaysia, Kubang Kerian, MYS 2. Pharmacology, School \\ of Medical Sciences, Universiti Sains Malaysia, Kubang Kerian, MYS 3. Microbiology and Parasitology, School of \\ Medical Sciences, Universiti Sains Malaysia, Kubang Kerian, MYS
}

Corresponding author: Rajendran Punitan, punitan1985@gmail.com

\section{Abstract}

\section{Introduction}

Pseudomonas aeruginosa is a common cause of microbial keratitis that can cause a significant loss of visual acuity. Antibiotics, including fluoroquinolones and aminoglycosides, are clinically effective against Pseudomonas-induced keratitis, but their effectiveness has been conspicuously reduced as resistant pathogens have become more potent. This study sought to evaluate the clinical and antibacterial effects of tualang honey as an alternative therapeutic agent against Pseudomonas-induced keratitis.

\section{Methods}

We conducted a randomized control trial in which 30 rabbits were injected intrastromally with 1,000 colonyforming units (CFU) of Pseudomonas aeruginosa in the right eye of each rabbit $(n=30)$. The rabbits were then randomized into three groups of 10 rabbits each. Group A was treated with topical gentamicin $0.3 \%$, group B was treated with topical tualang honey $30 \%$, and group C received both treatments. The specified treatments were administered every two hours from 24 to 48 hours post injection, and subsequently every four hours for six days. Clinical examinations were performed on days one, two, three, five, and seven, and the mean results of slit lamp examinations (SLEs) were documented. On day seven after pseudomonas induction, the rabbits were euthanized and their corneas were harvested to determine the median CFU per cornea.

\section{Results}

There were no statistically significant differences $(p>0.05)$ in mean SLE scores $(p=0.209)$ or median CFU values $(\mathrm{p}=0.820)$ between the three groups.

\section{Conclusion}

Received 02/22/2019

Review began 03/02/2019 Review ended 03/22/2019 Published 03/27/2019

() Copyright 2019

Punitan et al. This is an open access article distributed under the terms of the Creative Commons Attribution License CC-BY 3.0., which permits unrestricted use, distribution, and reproduction in any medium, provided the original author and source are credited.
Topical gentamicin, topical tualang honey, and the combination of the two all showed similar clinical and antimicrobial effects in treating Pseudomonas-induced keratitis in rabbits. These findings should be verified in further studies with larger sample sizes and the addition of a control group.

\section{Categories: Ophthalmology}

Keywords: tualang honey, pseudomonas aeruginosa, pseudomonas-induced keratitis

\section{Introduction}

Pseudomonas aeruginosa is a common cause of microbial keratitis that can cause a significant loss of visual acuity [1]. In contact lens users, Pseudomonas-induced keratitis can progress rapidly to corneal perforation within 24 hours [2-4]. Pharmaceutical treatments must therefore be effective at rapidly eradicating the organism. Fluoroquinolone and aminoglycoside antibiotics, including ceftazidime, gentamicin, ciprofloxacin, and combinations thereof, have been empirically shown to be effective and are the current standard treatment responses [5-6]. Intensive monotherapy is adequate if the corneal ulcer is small and peripheral with no impending perforation; but combination antibiotic therapy is recommended for more severe cases.

There have been recent reports of microbial resistance to gentamicin and ciprofloxacin in Australia, Europe, and North America [7-10]. As resistant pathogens become more common and more potent, antibiotics' effectiveness has been conspicuously reduced. Alternative antimicrobial strategies are therefore needed. This has prompted a number of studies that have reevaluated the clinical therapeutic benefits of ancient remedies, including honey, which is known to have antimicrobial and wound-healing properties [11-15]. 
Tualang honey is produced by the rock bee (apis dorsata), which builds hives high up in the branches of the tualang tree (kompassia excelsa or mengaris) in Malaysia. It is commonly used by locals as both a food and as a medicinal product. It has been demonstrated to have bactericidal and bacteriostatic properties against a wide range of bacteria [16-17], and has been shown to inhibit the growth of several bacterial strains, including Streptococcus pyogenes, Staphylococcus aureus, Salmonella typhi, coagulase-negative Staphylococcus spp, Escherichia coli, Pseudomonas aeruginosa, and Acinetobacter baumanii [18]. It also has anti-inflammatory and antioxidant properties that can facilitate wound healing [15, 19-20]. Patients have also reported that they prefer the tualang honey hydrogel dressings to conventional dressings because the treatment has a soothing effect, reduces discomfort during dressing changes, and provides a long-lasting pleasant odor [21].

The potency of tualang honey against microorganisms suggests its potential as an alternative therapeutic agent for certain medical conditions, especially wound infections [15, 19]. However, no experimental in-vivo study has been published that quantifies the effectiveness of tualang honey in treating infective eye conditions. The present study thus sought to analyze the clinical and antibacterial effects of tualang honey for treating Pseudomonas-induced keratitis in rabbit eyes.

\section{Materials And Methods \\ Materials and methods}

We conducted a randomized control animal experiment between September 2014 and September 2016 at the Animal Research and Service Centre of the Microbiology Laboratory and Pharmacological Laboratory at the Universiti Sains Malaysia (Health Campus, Kubang Kerian, Kelantan, Malaysia). The study was approved by the research and ethical committee at the School of Medical Sciences and the Animal Ethics Committee at the Universiti Sains Malaysia Health Campus. A preliminary pilot study was conducted to verify the feasibility of the methods and clinical results.

\section{Animals}

Three rabbits were used for the preliminary pilot study and 30 rabbits were used in the main study. All specimens were New Zealand white adult rabbits aged eight to 10 months that weighed 2.5-3.0 kg and had clear corneas prior to beginning the experiment. The rabbits were maintained and handled according to ethical guidelines for the treatment of animals. They were housed individually in stainless steel cages at a controlled temperature and humidity level and had a 12-hour light/dark cycle. Food (pellets) and water were provided ad libitum. Prior to inoculation with the bacteria, the rabbits were anesthetized with ketamine hydrochloride (100 mg/ml; $35 \mathrm{mg} / \mathrm{kg}$ ) and xylazine hydrochloride $(20 \mathrm{mg} / \mathrm{ml} ; 2.4 \mathrm{mg} / \mathrm{kg})$. A drop of proparacaine hydrochloride $0.5 \%$ was then topically applied to the right eye of each rabbit. They were euthanized on day seven after injection of pseudomonas aeruginosa. During euthanasia, they were first anesthetized and were then administered a lethal overdose of pentobarbital $(60 \mathrm{mg} / \mathrm{ml} ; 125 \mathrm{mg} / \mathrm{kg})$.

\section{Bacterial strain and infection}

Pseudomonas aeruginosa (strain 27853) were grown in tryptic soy agar (TSA) overnight at $37^{\circ} \mathrm{C}$. The overnight culture was then inoculated into fresh tryptic soy broth (TSB) $(1: 100)$ and grown at $37^{\circ} \mathrm{C}$ for 18 hours. The bacteria were then serially diluted in TSB to 10,000 colony-forming units (CFU) per $1 \mathrm{ml}$. The diluted bacteria were then plated on TSA and their number was verified with a quantitative bacterial count. The right cornea of each rabbit was injected intrastromally with 1,000 CFU of Pseudomonas aeruginosa in $0.1 \mathrm{ml}$ of TSB [22-24].

\section{Treatment regime}

In the preliminary study, the rabbits were injected with Pseudomonas aeruginosa and then treated with a single topical drop of saline eye drops every two hours from 24 to 48 hours after injection, and subsequently every four hours until day seven, at which point they were euthanized.

In the actual study, the rabbits were randomized into three groups of 10 rabbits each $(n=30)$. Group A was treated with topical gentamicin $0.3 \%$, group B was treated with topical tualang honey $30 \%$, and group C received both treatments. The specified treatments were administered every two hours from 24 to 48 hours after injection, and subsequently every four hours for six days. On day seven, post induction of Pseudomonas, all rabbits were euthanized and their corneas were harvested to determine the median CFU per cornea.

\section{Clinical eye examinations}

To evaluate pathological changes in the eyes, a single blinded examiner performed slit lamp examinations (SLEs) on all rabbit corneas on days one, two, three, five, and seven after the injection of Pseudomonas aeruginosa. The examinations were performed using hand-held portable slit-lamp biomicroscope, and the results were clinically recorded using an ulcer scoring system for corneal infiltrates, corneal ulcers, hypopyons, and corneal perforations (Table 1); the possible scores ranged from zero (normal) to a maximum 


\section{Cureus}

of four [25].

\begin{tabular}{|c|c|c|c|}
\hline Grade & \multicolumn{3}{|l|}{ Focus of infection } \\
\hline 0 & No focus of infection & 0 & \\
\hline \multirow{3}{*}{1} & \multirow{3}{*}{ Corneal Infiltrate } & 1.25 & Corneal infiltrate limited in the inoculated area \\
\hline & & 1.50 & Corneal infiltrate $\leq 1 / 2$ corneal thickness \\
\hline & & 1.75 & Corneal infiltrate $>1 / 2$ corneal thickness \\
\hline \multirow{3}{*}{2} & \multirow{3}{*}{ Corneal Ulcer } & 2.25 & Diameter $\leq 3 \mathrm{~mm}$ \\
\hline & & 2.50 & $>3 \mathrm{~mm}$ diameter $<5 \mathrm{~mm}$ \\
\hline & & 2.75 & Diameter $\geq 5 \mathrm{~mm}$ \\
\hline \multirow{3}{*}{3} & \multirow{3}{*}{ Hypopyon } & 3.25 & Altitude $\leq 1 / 3$ Anterior chamber (AC) \\
\hline & & 3.50 & $>1 / 3 \mathrm{AC}$ altitude $<1 / 2 \mathrm{AC}$ \\
\hline & & 3.75 & Altitude $\geq 1 / 2 \quad A C$ \\
\hline 4 & Corneal perforation & 4.00 & \\
\hline
\end{tabular}

\section{TABLE 1: Slit Lamp Examination (SLE) scores}

Adapted from Dong et al. [25].

\section{CFU determination}

On day seven, after the injection of pseudomonas aeruginosa, the rabbits were anesthetized and then euthanized. Their corneas were harvested and homogenized in sterile phosphate buffer and the homogenates were serially diluted (1:10) in fresh TSB, plated in triplicate TSA, and incubated at $37^{\circ} \mathrm{C}$ overnight. The bacterial colonies were then counted to determine the median CFU per cornea for each treatment group.

\section{Statistical analysis}

The data were analyzed using repeated measures multivariate ANOVA in Statistical Package for Social Sciences (IBM, Armonk, NY). Abnormally distributed data were analyzed using the Kruskal Wallis test. Values were expressed as medians (interquartile range). We considered $\mathrm{p}$ values of $<0.05$ to be statistically significant.

\section{Results}

\section{Preliminary study (Phase I)}

Clinical Anti-inflammatory Effects

The mean SLE results of the preliminary study are shown in Table 2. The mean SLE scores increased from day one to day seven post bacterial injection.

\begin{tabular}{|c|c|c|c|c|c|}
\hline \multirow{2}{*}{ Group Time } & \multicolumn{5}{|c|}{ Mean SLE score (SD) } \\
\hline & Day 1 & Day 2 & Day 3 & Day 5 & Day 7 \\
\hline Preliminary & $1.67(0.14)$ & $2.42(0.72)$ & $3.00(0.43)$ & $3.33(0.14)$ & $3.58(0.14)$ \\
\hline
\end{tabular}

TABLE 2: Mean slit lamp examination (SLE) scores over time in the preliminary study $(n=3)$ 


\section{Cureus}

All three rabbits showed severe clinical conjunctival hyperemia and corneal edema that progressively worsened over time (Figure 1).

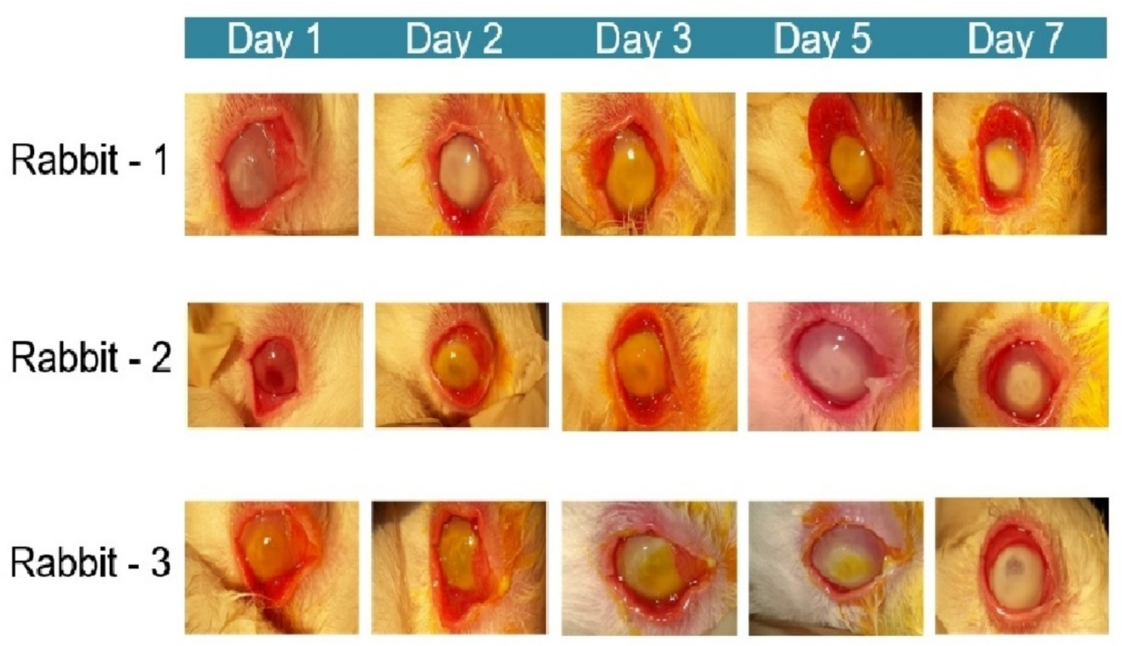

FIGURE 1: Conjunctival hyperemia and corneal edema in rabbits with Pseudomonas-induced keratitis in preliminary study

Table 3 shows the mean CFU count of their corneas on day seven. Although no bacterial growth was observed in rabbits one and three, this was probably due to an inaccurate calculation method of mean CFU; this method was improved for the actual study.

\begin{tabular}{|l|l|}
\hline Rabbit & CFU count \\
\hline 1 & No growth \\
\hline 2 & 1070 \\
\hline 3 & No growth \\
\hline
\end{tabular}

TABLE 3: Mean colony forming unit (CFU) of rabbits in the preliminary study on day seven after infection with Pseudomonas aeruginosa

SD: Standard deviation; CFU: colony forming unit

\section{Actual study (Phase II)}

Clinical Anti-inflammatory Effects

In all three groups, all of the rabbits' eyes demonstrated severe clinical inflammatory features (conjunctival hyperemia and corneal edema) on day one post infection with Pseudomonas aeruginosa. In all three groups, the conjunctival hyperemia improved progressively from day one until day seven, while the corneal edema worsened from day one until day three and then remained the same until day seven. The observed clinical features of both conjunctival hyperemia and corneal edema in all three different treatment groups are shown in Figure 2. 


\section{Cureus}

Day $1 \quad$ Day $2 \quad$ Day $3 \quad$ Day $5 \quad$ Day 7

Group A
Rabbit - 1
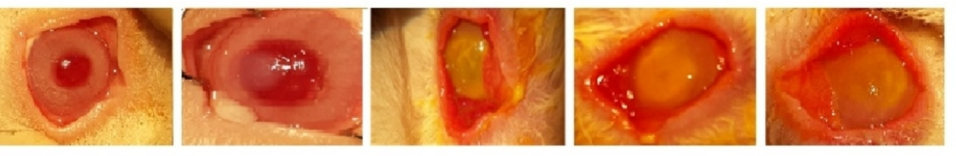

Group B

Rabbit - 1
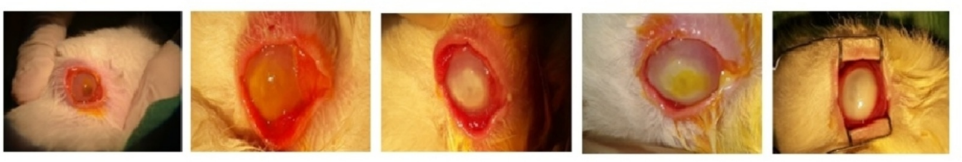

Group C

Rabbit - 1
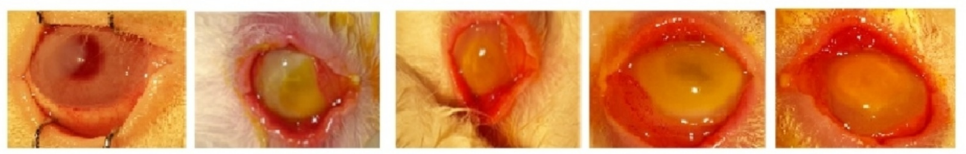

FIGURE 2: Conjunctival hyperemia and corneal edema in rabbits with Pseudomonas-induced keratitis in the actual study

\section{SLE scores}

Table 4 shows the total mean SLE scores for each treatment group. The scores were almost identical between group A (gentamicin) and group C (both gentamicin and tualang honey), and there were no statistically significant differences between any of the three groups $(\mathrm{p}=0.209)$.

\begin{tabular}{|c|c|c|c|}
\hline Group & Mean difference $(95 \% \mathrm{Cl})$ & F statistic (df 1, df 2) & p value \\
\hline$A$ & $2.99(2.86,3.11)$ & \multirow{3}{*}{$1.66(2,27)$} & \multirow{3}{*}{0.209} \\
\hline$B$ & $2.88(2.75,3.01)$ & & \\
\hline $\mathrm{C}$ & $3.04(2.91,3.17)$ & & \\
\hline
\end{tabular}

\section{TABLE 4: Total mean slit lamp examination (SLE) scores of each treatment group}

A: Gentamicin $0.3 \%$ treated group; B: Tualang honey 30\% treated group; C: Combination of gentamicin $0.3 \%$ and tualang honey $30 \%$ treated groups; df: degree of freedom. Repeated measure ANOVA adjustment by Bonferroni correction, $p$-value $<0.05$ (significant)

Table 5 presents the mean SLE scores of each treatment group at different time points. The mean SLE scores in all three groups increased progressively over the study period. Notably, on day five, there was a significant difference in the mean SLE scores of groups B (tualang honey) and C (both gentamicin and tualang honey). 


\section{Cureus}

\begin{tabular}{|l|lllll|}
\hline \multicolumn{7}{|l}{ Mean SLE score (SD) } & & \\
\hline & Day 1 & Day 2 & Day 3 & Day 5 & Day 7 \\
\hline A & $2.28(0.55)$ & $2.89(0.16)$ & $3.06(0.11)$ & $3.15(0.12)$ & $3.60(0.12)$ \\
B & $2.33(0.55)$ & $3.03(0.13)$ & $2.83(0.12)$ & $2.83(0.11)$ & $3.40(0.18)$ \\
C & $2.25(0.55)$ & $3.13(0.13)$ & $3.18(0.18)$ & $3.33(0.11)$ & $3.33(0.12)$ \\
$P(A):(B)$ & $>0.95$ & $>0.95$ & 0.711 & 0.118 & 0.709 \\
$P(A):(C)$ & $>0.95$ & 0.510 & $>0.95$ & 0.761 & 0.322 \\
$P(B):(C)$ & $>0.95$ & $>0.95$ & 0.131 & 0.008 & $>0.95$ \\
\hline
\end{tabular}

\section{TABLE 5: Mean slit lamp examination (SLE) scores of each treatment group over time}

A: Gentamicin $0.3 \%$ treated group; B: Tualang honey $30 \%$ treated group; C: Combination of gentamicin $0.3 \%$ and tualang honey $30 \%$ treated groups. Repeated measures ANOVA within group analysis with $95 \%$ confidence interval adjustment by Bonferroni correction, $p$-value $<0.05$ (significant) with $F$ statistic $1.66(2,27)$

\section{Antimicrobial effects}

The bacterial CFU count of each treatment group on day seven post infection is shown in Table 6. Group C (both gentamicin and tualang honey) had the lowest median CFU count (48), while Group B (tualang honey) had the highest. However, the differences between the three groups were not statistically significant $(\mathrm{p}=0.82)$.

\begin{tabular}{|l|l|l|}
\hline Groups & Median CFU (IQR) & Chi square statistic (df) \\
\hline A & $280(899)$ & \\
\hline B & $699(835)$ & $4.993(2)$ \\
\hline C & $48(1353)$ & \\
\hline
\end{tabular}

TABLE 6: Median colony forming unit (CFU) of rabbits in each treatment group on day seven after infection with Pseudomonas aeruginosa

A: Gentamicin $0.3 \%$ treated group; B: Tualang honey $30 \%$ treated group; C: Combination of gentamicin $0.3 \%$ and tualang honey $30 \%$ treated groups; df: degree of freedom; IQR: interquartile range. Kruskal Wallis test, p-value $<0.05$ (significant)

\section{Discussion}

Multiple studies across a variety of disciplines have reported on the uses of tualang honey [21,26-27], and more particularly on its use as a treatment for a variety of eye diseases [26,28]. Tan et al. observed its antibacterial effects against Pseudomonas aeruginosa isolates at concentrations of $\geqslant 25 \%$ [19], while Cernak et al. found that its antimicrobial properties could be used to prevent corneal scarring caused by infections [28]. Other studies have found that its antioxidant properties neutralize free radicals and gives it an antiinflammatory effect [15-17], which can expedite treatment of blepharitis and keratitis [29].

In the present study, we found no statistically significant differences in the total mean SLE scores of the groups treated with tualang honey versus gentamicin or both $(p>0.209)$. The mean SLE scores in all three groups increased from day one to day seven post-infection, which suggests ongoing inflammatory processes during the early phase of infection. This is consistent with a study performed by Dong et al. that reported increasing inflammation from days four to ten in the eyes of infected rabbits treated with topical natamycin and natacyn, with subsequent inflammation regression until the end of the study on day 21 [25].

We also observed similar clinical responses to Pseudomonas-induced keratitis with all three treatment groups during the study period. These findings are consistent with Bashkaran et al, who found that the honey-treated group was as good as the conventionally treated group in terms of the healing process of an alkali-induced corneal epithelial defect in rabbits [30]. However, we were not able to find any other similar studies. 
We observed no statistically significant differences in the median CFUs on day seven of our study ( $p>0.820$ ). However, we found that there was a similar treatment benefit in all three groups with a reduction of median CFU on day seven. This finding is inconsistent with the study done by Nejabat et al. who found that topical $90 \%$ concentrated natural honey was equally effective as ciprofloxacin $0.3 \%$ at treating Pseudomonasinduced keratitis in rabbit eyes and that both treatments led to a significant reduction in the number of bacteria compared to controls [23].

The present study was limited by small sample size and did not include a control group due to financial constraints. Furthermore, we used tualang honey 30\%, and while this is above the minimum tested bactericidal concentration of $25 \%$ for Pseudomonas aeruginosa isolates [16], it is plausible that more concentrated tualang honey might yield better results. Finally, although the rabbit intrastromal injection model is widely used and accepted as an in-vivo keratitis model for comparison to human eye infections, and while the rabbits in the present study were standardized in regard to age, gender, size, and source, the possibility always remains that the immune response observed in the rabbits here may not be generalizable to other rabbit or human populations due to genetic variations [24].

\section{Conclusions}

Topical gentamicin, topical tualang honey, and a combination of the two all demonstrated similar clinical and antimicrobial effects in treating Pseudomonas-induced keratitis in rabbits. Additional research with larger sample sizes and the addition of a control group are warranted to further explore the antibacterial effects of tualang honey in Pseudomonas-induced keratitis.

\section{Additional Information \\ Disclosures}

Human subjects: All authors have confirmed that this study did not involve human participants or tissue. Animal subjects: Animal ethics committee USM Issued protocol number USM animal ethics approval 2014/(91)(575) . Conflicts of interest: In compliance with the ICMJE uniform disclosure form, all authors declare the following: Payment/services info: Research University Grant 1001/PPSP/812104. Financial relationships: All authors have declared that they have no financial relationships at present or within the previous three years with any organizations that might have an interest in the submitted work. Other relationships: All authors have declared that there are no other relationships or activities that could appear to have influenced the submitted work.

\section{References}

1. Jeng BH, McLeod SD: Microbial keratitis. Br J Ophthalmol. 2003, 87:805-806. 10.1136/bjo.87.7.805

2. Bourcier T, Thomas F, Borderie V, Chaumeil C, Laroche L: Bacterial keratitis: predisposing factors, clinical and microbiological review of 300 cases. The Br J Ophthalmol. 2003, 87:834-838. 10.1136/bjo.87.7.834

3. Stapleton F, Dart JK, Seal DV, Matheson M: Epidemiology of Pseudomonas aeruginosa keratitis in contact lens wearers. Epidemiol Infect. 1995, 114:395-402. 10.1017/S0950268800052109

4. Fong CF, Tseng CH, Hu FR, Wang IJ, Chen WL, Hou YC: Clinical characteristics of microbial keratitis in a university hospital in Taiwan. Am J Ophthalmolol. 2004, 137:329-336. 10.1016/j.ajo.2003.09.001

5. Jones DB: Initial therapy of suspected microbial corneal ulcers. Sur Ophthalmol. 1979, 24:105-116. 10.1016/0039-6257(79)90128-0

6. O'Brien TP: Management of bacterial keratitis: beyond exorcism towards consideration of organism and host factors. Eye (London, England. 2003, 17:957-974. 10.1038/sj.eye.6700635

7. Ly CN, Pham JN, Badenoch PR, Bell SM, Hawkins G, Rafferty DL, McClellan KA: Bacteria commonly isolated from keratitis specimens retain antibiotic susceptibility to fluoroquinolones and gentamicin plus cephalothin. Clin Experiment ophthalmol. 2006, 34:44-50. 10.1111/j.1442-9071.2006.01143.x

8. Green M, Apel A, Stapleton F: Risk factors and causative organisms in microbial keratitis . Cornea. 2008, 27:22-27. 10.1097/ICO.0b013e318156caf2

9. Willcox MDP: Management and treatment of contact lens-related Pseudomonas keratitis . Clin Ophthalmol (Auckland, NZ). 2012, 6:919-924. 10.2147/OPTH.S25168

10. Lichtinger A, Yeung SN, Kim P, et al.: Shifting trends in bacterial keratitis in Toronto: an 11-year review . Ophthalmol. 2012, 119:1785-1790. 10.1016/j.ophtha.2012.03.031

11. Basualdo C, Sgroy V, Finola MS, Marioli JM: Comparison of the antibacterial activity of honey from different provenance against bacteria usually isolated from skin wounds. Vet microbiol. 2007, 124:375-381. 10.1016/j.vetmic.2007.04.039

12. Mandal S, DebMandal M, Pal NK, Saha K: Synergistic anti-staphylococcus aureus activity of amoxicillin in combination with emblica officinalis and nymphae odorata extracts. Asian Pac J Trop Med. 2010, 3:711-714. 10.1016/S1995-7645(10)60171-X

13. Mandal S, DebMandal M, Pal NK, Saha K: Antibacterial activity of honey against clinical isolates of escherichia coli, pseudomonas aeruginosa and salmonella enterica serovar Typhi. Asian Pac J Trop Med. 2010, 3:961-964. 10.1016/S1995-7645(11)60009-6

14. Khoo YT, Halim AS, Singh KKB, Mohamad NA: Wound contraction effects and antibacterial properties of Tualang honey on full-thickness burn wounds in rats in comparison to hydrofibre. BMC Complement Altern Med. 2010, 10. 10.1186/1472-6882-10-48

15. Zainol MI, Mohd Yusoff K, Mohd Yusof MY: Antibacterial activity of selected Malaysian honey . BMC Complement Altern Med. 2013, 13:129. 10.1186/1472-6882-13-129 
16. Tan HT, Rahman RA, Gan SH, Halim AS, Hassan SA, Sulaiman SA, Bs KK: The antibacterial properties of Malaysian tualang honey against wound and enteric microorganisms in comparison to manuka honey. BMC Complement Alterna Med. 2009, 9:34-34. 10.1186/1472-6882-9-34

17. Mohamed Z, Alfarisi H: Tualang honey: composition, physiochemical properties and clinical importance . Int Res J Pharm. 2017, 8:1-5. 10.7897/2230-8407.089150

18. Nasir N-AM, Halim AS, Singh K-KB, Dorai AA, Haneef M-NM: Antibacterial properties of tualang honey and its effect in burn wound management: a comparative study. BMC Complement Altern Med. 2010, 10:1-7. 10.1186/1472-6882-10-31

19. Tan HZ, Abdul Rahman R, Gan SH, Halim AS, Hassan SA: The antibacterial properties of Malaysian tualang honey against wound and enteric microorganisms in comparison to manuka honey. BMC Complement Altern Med. 2009, 9. 10.1186/1472-6882-9-34

20. Ahmed S, Othman NH: Review of the medicinal effects of tualang honey and a comparison with manuka honey. Malay J Med Sci. 2013, 20:6-13. Accessed: May 2013: https://www.ncbi.nlm.nih.gov/pubmed/23966819.

21. Imran FH, Dorai AA, Halim AS, Sulaiman WAW: Tualang honey hydrogel in the treatment of split-skin graft donor sites. J ApiPro ApiMed Sci. 2011, 3:33-37. 10.3896/IBRA.4.03.1.06

22. Thibodeaux BA, Dajcs JJ, Caballero AR, Marquart ME, Girgis DO, O'Callaghan RJ: Quantitative comparison of fluoroquinolone therapies of experimental gram-negative bacterial keratitis. Curr Eye Res. 2004, 28:337342. 10.1076/ceyr.28.5.337.28676

23. Nejabat M, Astaneh A, Eghtedari M, Mosallaei M, Ashraf MJ, Mehrabani D: Effect of honey in pseudomonas aeruginosa induced stromal keratitis in rabbits. J Appl Anim Res. 2009, 35:101-104. 10.1080/09712119.2009.9706996

24. McCormick C, Caballero A, Tang A, Balzli C, Song J, O'Callaghan R: Effectiveness of a new tobramycin (0.3\%) and dexamethasone $(0.05 \%)$ formulation in the treatment of experimental Pseudomonas keratitis. Curr Med Res Opin. 2008, 24:1569-1575. 10.1185/03007990802079877

25. Dong XH, Gao WJ, He XP: Antifungal efficacy of natamycin in experimental fusarium solani keratitis . Int J Ophthalmol. 2012, 5:143.10.3980/j.issn.2222-3959.2012.02.05

26. Ahmed AK, Hoekstra MJ, Hage JJ, Karim RB: Honey-medicated dressing: transformation of an ancient remedy into modern therapy. Ann Plast Surg. 2003, 50:143-147. 10.1097/01.sap.0000032306.44107.c1

27. Othman Z, Shafin N, Zakaria R, Hussain NH, Mohammad WM: Improvement in immediate memory after 16 weeks of tualang honey (Agro Mas) supplement in healthy postmenopausal women. Menopause (New York, NY). 2011, 18:1219-1224. 10.1097/gme.0b013e31821e2044

28. Cernak M, Majtanova N, Cernak A, Majtan J: Honey prophylaxis reduces the risk of endophthalmitis during perioperative period of eye surgery. Phytother Res. 2012, 26:613-616. 10.1002/ptr.3606

29. Salehi A, Jabarzare S, Neurmohamadi M, Kheiri S, Rafieian-Kopaei M: A double blind clinical trial on the efficacy of honey drop in vernal keratoconjunctivitis. Evid Based Complement Alternat Med. 2014, 2014:4. $10.1155 / 2014 / 287540$

30. Bashkaran K, Zunaina E, Bakiah S, Sulaiman SA, Sirajudeen K, Naik V: Anti-inflammatory and antioxidant effects of Tualang honey in alkali injury on the eyes of rabbits: experimental animal study. BMC Complement Altern Med. 2011, 11:90. 10.1186/1472-6882-11-90 